\title{
Schwarzian derivative \\ and \\ Numata Finsler structures
}

\author{
C. DUVAL $\ddagger$
}

Centre de Physique Théorique, CNRS, Luminy, Case 907

F-13288 Marseille Cedex 9 (France) ${ }^{\S}$

\begin{abstract}
The flag curvature of the Numata Finsler structures is shown to admit a nontrivial prolongation to the one-dimensional case, revealing an unexpected link with the Schwarzian derivative of the diffeomorphisms associated with these Finsler structures.
\end{abstract}

Mathematics Subject Classification 2000: 58B20, 53A55

\section{$1 \quad$ Finsler structures in a nutshell}

\subsection{Finsler metrics}

A Finsler structure is a pair $(M, F)$ where $M$ is a smooth, $n$-dimensional, manifold and $F: T M \rightarrow \mathbb{R}^{+}$a given function whose restriction to the slit tangent bundle $T M \backslash M=\left\{(x, y) \in T M \mid y \in T_{x} M \backslash\{0\}\right\}$ is strictly positive, smooth, and positively homogeneous of degree one, i.e., $F(x, \lambda y)=\lambda F(x, y)$ for all $\lambda>0$; one furthermore demands that the $n \times n$ vertical Hessian matrix with entries $\mathrm{g}_{i j}(x, y)=\left(\frac{1}{2} F^{2}\right)_{y^{i} y^{j}}$ be positive definite, $\left(\mathrm{g}_{i j}\right)>0$. See [1]. These quantities are (positively) homogeneous of degree zero, and the fundamental tensor

$$
\mathrm{g}=\mathrm{g}_{i j}(x, y) d x^{i} \otimes d x^{j}
$$

defines a sphere's worth of Riemannian metrics on each $T_{x} M$ parametrized by the direction of $y$. See [2].

The distinguished "vector field"

$$
\ell=\ell^{i} \frac{\partial}{\partial x^{i}}, \quad \text { where } \quad \ell^{i}(x, y)=\frac{y^{i}}{F(x, y)},
$$

actually a section of $\pi^{*}(T M)$ where $\pi: T M \backslash M \rightarrow M$ is the natural projection, is such that $\mathrm{g}(\ell, \ell)=1$.

\footnotetext{
${ }^{\ddagger}$ mailto: duval@cpt.univ-mrs.fr

§UMR 6207 du CNRS associée aux Universités d'Aix-Marseille I et II et Université du Sud Toulon-Var; Laboratoire affilié à la FRUMAM-FR2291
} 
There is a wealth of Finsler structures, apart from the special case of Riemannian structures $(M, \mathrm{~g})$ for which $F(x, y)=\sqrt{\mathrm{g}_{i j}(x) y^{i} y^{j}}$. For instance, the so-called Randers metrics

$$
F(x, y)=\sqrt{a_{i j}(x) y^{i} y^{j}}+b_{i}(x) y^{i}
$$

satisfy all previous requirements if $a=a_{i j}(x) d x^{i} \otimes d x^{j}$ is a Riemann metric and if the 1-form $b=b_{i}(x) d x^{i}$ is such that $a^{i j}(x) b_{i}(x) b_{j}(x)<1$ for all $x \in M$.

\subsection{Flag curvature}

Unlike the Riemannian case, there is no canonical linear Finsler connection on $\pi^{*}(T M)$. An example, though, is provided by the Chern connection $\omega_{j}^{i}=\Gamma_{j k}^{i}(x, y) d x^{k}$ which is uniquely defined by the following requirements [1]: (i) it is symmetric, $\Gamma_{j k}^{i}=\Gamma_{k j}^{i}$, and (ii) it almost transports the metric tensor, i.e., $d g_{i j}-\omega_{i}^{k} g_{j k}-\omega_{j}^{k} g_{i k}=2 C_{i j k} \delta y^{k}$, with $\delta y^{i}=d y^{i}+N_{j}^{i} d x^{j}$, where the $N_{j}^{i}(x, y)=\Gamma_{j k}^{i} y^{k}$ are the components of the non linear connection associated with the Chern connection, and the $C_{i j k}(x, y)=\frac{1}{2}\left(\mathrm{~g}_{i j}\right)_{y^{k}}$ those of the Cartan tensor, specific to Finsler geometry.

Using the "horizontal covariant derivatives" $\delta / \delta x^{i}=\partial / \partial x^{i}-N_{i}^{j} \partial / \partial y^{j}$, one expresses the (horizontal-horizontal part of the) Chern curvature by

$$
R_{j k l}^{i}=\frac{\delta}{\delta x^{k}} \Gamma_{j l}^{i}+\Gamma_{m k}^{i} \Gamma_{j l}^{m}-(k \leftrightarrow l),
$$

and the flag curvature (associated with the flag $\ell \wedge v$ defined by $v \in T_{x} M$ ) by

$$
K(x, y, v)=\frac{R_{i k} v^{i} v^{k}}{\mathrm{~g}(v, v)-\mathrm{g}(\ell, v)^{2}}, \quad \text { where } \quad R_{i k}=\ell^{j} R_{j i k \ell} \ell^{\ell} .
$$

One says that a Finsler structure is of scalar curvature if $K(x, y, v)$ does not depend on the vector $v$, i.e., if

$$
R_{i k}=K(x, y) h_{i k},
$$

with $h_{i k}=\mathrm{g}_{i k}-\ell_{i} \ell_{k}$ the components of the "angular metric", where $\ell_{i}=\mathrm{g}_{i j} \ell^{j}\left(=F_{y^{i}}\right)$. See [1, 2].

\section{$2 \quad$ Numata Finsler structures}

\subsection{The Numata metric}

Numata [4 has proved that metrics of the form $F(x, y)=\sqrt{q_{i j}(y) y^{i} y^{j}}+b_{i}(x) y^{i}$, on $T M$ where $M \subset \mathbb{R}^{n}$, with $\left(q_{i j}\right)>0$ and $d b=0$ are, indeed, of scalar curvature. See [2].

Of some interest is the special case $q_{i j}=\delta_{i j}$ and $b=d f$ with $f \in C^{\infty}(M)$, viz.,

$$
F(x, y)=\sqrt{\delta_{i j} y^{i} y^{j}}+f_{x^{i}} y^{i}
$$

where

$$
M=\left\{x \in \mathbb{R}^{n} \mid \sum_{i=1}^{n} f_{x^{i}}^{2}<1\right\}
$$


The computation of the flag curvature of this particular Randers metric (1.3) can be found in [1] and yields

$$
K(x, y)=\frac{3}{4} \frac{1}{F^{4}}\left(f_{x^{i} x^{j}} y^{i} y^{j}\right)^{2}-\frac{1}{2} \frac{1}{F^{3}} f_{x^{i} x^{j} x^{k}} y^{i} y^{j} y^{k} .
$$

\subsection{Flag curvature \& Schwarzian derivative}

The expression (2.3) of the flag curvature of the Numata metric (2.1) holds for $n \geq 2$.

If $n=1$, the left-hand side of (1.6) vanishes along with the curvature (1.4), while its right-hand vanishes as well since the angular metric has rank zero. For this particular dimension, Equation (1.6) trivially holds true, but tells, however, nothing about the flag curvature $K(x, y)$.

At this stage, it is worth noting that (2.3) indeed admits a prolongation to the one-dimensional case; it is therefore tempting to specialize its expression for $n=1$.

Suppose, thus, that $M \subset S^{1}$ is a nonempty open subset (2.2), so that we have $T M \backslash M=T_{+} M \sqcup T_{-} M$, where $T_{ \pm} M=M \times \mathbb{R}_{*}^{ \pm}$. The metric (2.1) then reads

$$
F(x, y)=|y|+f^{\prime}(x) y
$$

using an affine coordinate, $x$, on $S^{1}$, with $-1<f^{\prime}(x)<+1$ (see (2.2)); its restrictions to $T_{ \pm} M$ are given by $F_{ \pm}(x, y)=\varphi_{ \pm}^{\prime}(x) y>0$, where

$$
\varphi_{ \pm}^{\prime}(x)=f^{\prime}(x) \pm 1
$$

implying $\varphi_{ \pm} \in \operatorname{Diff}_{ \pm}\left(S^{1}\right)$, with $\left|\varphi_{ \pm}^{\prime}(x)\right|<2($ all $x \in M)$.

The Numata metric (2.4) on $T_{+} M$, say, is thus associated, via (2.5), to orientationpreserving diffeomorphisms $\varphi$ of $S^{1}$ such that $0<\varphi^{\prime}(x)<2$ (all $x \in M$ ). Given such a $\varphi \in \operatorname{Diff}_{+}\left(S^{1}\right)$, the fundamental tensor (1.1) retains the form $\mathrm{g}=\varphi^{\prime}(x)^{2} d x^{2}$ and is, naturally, a Riemannian metric on $M$.

Rewriting Equation (2.3) for $T_{+} M$, and bearing in mind that $y=F(x, y) / \varphi^{\prime}(x)$, we readily find that $K(x, y)$ is actually independent of $y$, namely

$$
K(x)=-\frac{1}{2} \frac{1}{\varphi^{\prime}(x)^{2}} S(\varphi)(x),
$$

where

$$
S(\varphi)(x)=\frac{\varphi^{\prime \prime \prime}(x)}{\varphi^{\prime}(x)}-\frac{3}{2}\left(\frac{\varphi^{\prime \prime}(x)}{\varphi^{\prime}(x)}\right)^{2}
$$

denotes the Schwarzian derivative [5] of the diffeomorphism $\varphi$ of $S^{1}$. The argument clearly still holds, mutatis mutandis, for orientation-reversing diffeomorphisms of $S^{1}$. 
We have thus proved the

Theorem 2.1. The Numata Finsler structure $(M, F)$, with metric $F$ given by (2.4) where $M \subset S^{1}$ is defined by 2.9), induces a Riemannian metric, $\mathrm{g}(\varphi)=\varphi^{*}\left(d x^{2}\right)$, where $\varphi \in \operatorname{Diff}\left(S^{1}\right)$ is as in 2.5. The flag curvature (2.3) admits a prolongation to this one-dimensional case and retains the form

$$
K=-\frac{1}{2} \frac{\mathrm{S}(\varphi)}{\mathrm{g}(\varphi)},
$$

where $\mathrm{S}(\varphi)=S(\varphi)(x) d x^{2}$ is the Schwarzian quadratic differential of $\varphi \in \operatorname{Diff}\left(S^{1}\right)$.

As an illustration, the one-dimensional Numata Finsler structures of constant flag curvature are associated, through (2.5), to the solutions $\varphi$ of (2.8) for $K \in \mathbb{R}$, viz., $\varphi_{ \pm}(x)=K^{-\frac{1}{2}} \arctan \left(K^{\frac{1}{2}}(a x+b) /(c x+d)\right)$ where $a, b, c, d \in \mathbb{R}$ with $a d-b c= \pm 1$.

Let us mention another instance where the Schwarzian derivative is associated with curvature, namely the geometry of curves in Lorentzian surfaces of constant curvature [3].

Discussions with P. Foulon are warmly acknowledged.

\section{References}

[1] D. Bao, S.-S. Chern, and Z. Shen, An Introduction to Riemann-Finsler Geometry, GTM 200, Springer, New York, 2004.

[2] D. Bao, and C. Robles, "Ricci and Flag Curvatures in Finsler Geometry", in A Sampler of Riemann-Finsler Geometry, D. Bao, R. L. Bryant, S.-S. Chern, and Z. Shen (Editors), MSRI Publications 50, Cambridge University Press, 2004.

[3] C. Duval, and V. Ovsienko, "Lorentzian Worldlines and the Schwarzian Derivative", Funct. Anal. Applic. 34:2 (2000), 135-137.

[4] S. Numata, "On the torsion tensors $R_{j h k}$ and $P_{h j k}$ of Finsler spaces with a metric $d s=\left(g_{i j}(d x) d x^{i} d x^{j}\right)^{1 / 2}+b_{i}(x) d x^{i} "$, Tensor (N.S.) 32 (1978), 27-32.

[5] V. Ovsienko, and S. Tabachnikov, Projective Differential Geometry Old And New: From The Schwarzian Derivative To The Cohomology Of Diffeomorphism Group, Cambridge University Press, 2005, and References therein. 